\title{
The importance of data sharing
}

It has long been understood that scientific research is the path adequately solving population health issues. This includes a wide range of different scenarios where human health is appraised: diagnosis, treatment, quality, professional education, etc.

In addition to such essential contribution, research also helps to develop a healthy critical stance, which will both contribute to making better decisions for our patients and have a positive effect on other aspects of our lives.

It is worth noting that reports of such relevant activity are ultimately a "sworn statement." The value of such "sworn statement" lies exclusively on the scientific community's trust in investigators.

If you take a moment to think about the most recent original article you have read, you will certainly remember that, in their results, the author is describing the assessment of a specific number of patients who, after certain exposure, responded in a specific manner. We have to admit that we have not seen any of the patients mentioned by the investigator and, let alone, had a chance to assess their behavior after the studied exposure. We simply trust in what the author states.

In spite of the altruism generally involved in any research, undoubtedly there may be conflicts of interest, and we cannot completely overlook the possibility of unacceptable biases, or fraud even. Over time, the scientific community has developed mechanisms to avoid, or at least reduce, such possibility.

The first line of defense in research integrity is made up by research ethics committees. The requirement that investigations have to be previously assessed and approved by such committee warrants both subject protection and an adequate management of conflicts of interest, if any.

Then, scientific publications added the requirement of prior registration of the research protocols. Such requirement was originally designed to avoid the bias of publishing only the positive outcomes of drug research and soon extended to other types of studies and warrants research transparency in general.

After these initial barriers, peer review takes its position. Although it is widely criticized and an admittedly imperfect method, it is still the best mechanism available to select the investigations that will be supported through scientific publication.

More recently, a new line of defense has been introduced to improve scientific research transparency: data sharing.

Data sharing is the practice of making unprocessed data ("raw data") used for research available to other investigators. Data sharing increases research transparency by allowing to confirm the results interpretation. It also maximizes data usefulness by allowing their use in other investigations. ${ }^{1}$

As any other research component, data sharing should also be considered in the research protocol. On the one side, for data to be shared, they should meet certain characteristics known as the FAIR Principles (Findable, Accessible, Interoperable, Reusable). ${ }^{2}$ On the other side, the plan for data sharing should also consider which data will be shared, who will access them, where they will be stored, when they will be shared, and how they will be accessed. These items are usually described in the data sharing statement required by journals for manuscript submission. The International Committee of Medical Journal Editors (ICMJE) provides examples of how to comply with this statement policy. ${ }^{3}$

Although the data sharing initiative was initially designed for clinical research, ${ }^{4}$ now it is considered convenient to extend it to all types of research, including observational studies. ${ }^{5}$

Like any new initiative, data sharing has also given rise to controversy. There is concern in relation to the confidentiality and privacy of research participants. There is also a risk that secondary data analysis, out of the context in which they were obtained, may easily lead to unreliable results in secondary research. Finally, the fact that "primary" investigators' activity may be threatened by those who use their data in a secondary manner is also a concern because they may gain a greater visibility and prestige. ${ }^{6}$ Although the aspects mentioned above are still a matter of debate, the former may be discussed with an ethics committee, whereas the latter may be settled via the appropriate recognition of the primary investigator by the users of shared data.

Scientific journals have the critical 
responsibility of safeguarding the integrity of investigations whose data are published. The requirement that investigations have to be approved by an ethics committee and registered in a public registry before initiation, the commitment to share data and peer review, are relevant to support such commitment.

Archivos Argentinos de Pediatría adheres to these practices in an effort to offer high quality scientific evidence and a greater level of transparency.

\section{Fernando Ferrero}

Editor

http: / / dx.doi.org/10.5546/ aap.2022.eng.74

To cite: Ferrero F. The importance of data sharing. Arch Argent Pediatr 2022;120(2):74-75.

\section{REFERENCES}

1. Sixto-Costoya A, Aleixandre-Benavent R, Vidal-Infer A, Lucas-Domínguez R, Castelló-Cogollos L. Data sharing: qué son y cómo se pueden compartir los datos de investigación. Manual de recomendación para gestores de la información. Sociedad Española de Documentación e Información Científica, 2019. [Accessed on:November $\left.24^{\text {th }}, 2021\right]$. Available at: https: / / www.sedic. es/wp-content/uploads/2020/01/Data_Sharing-DEF.pdf

2. Wilkinson MD, Dumontier M, Aalbersberg IJ, Appleton G, et al. The FAIR Guiding Principles for scientific data management and stewardship. Sci Data. 2016;3:160018.

3. International Committee of Medical Journal Editors. Recommendations. Data sharing. [Accessed on: November $\left.24^{\text {th }}, 2021\right]$. Available at: http://www.icmje. org/recommendations/browse/publishing-and-editorialissues / clinical-trial-registration.html\#: : :text=Data $\% 20$ sharing $\% 20$ statements $\% 20$ must $\% 20$ indicate,(e.g. $\% 2 \mathrm{C} \% 20$ study $\% 20$ protocol $\% 2 \mathrm{C} \% 20$ statistical

4. Committee on Strategies for Responsible Sharing of Clinical Trial Data; Board on Health Sciences Policy; Institute of Medicine. Sharing Clinical Trial Data: Maximizing Benefits, Minimizing Risk. Washington (DC): National Academies Press (US); April 20, 2015.

5. Ewers M, Ioannidis JPA, Plesnila N. Access to data from clinical trials in the COVID-19 crisis: open, flexible, and time-sensitive. J Clin Epidemiol. 2021;130:143-6.

6. Bull S, Cheah PY, Denny S, Jao I, et al. Best practices for ethical sharing of individual-level health research data from low- and middle-income settings. J Empir Res Hum Res Ethics. 2015;10(3):302-13. 